\title{
Solution to Hofmeister effect challenge
}

\section{Mahdi Hashemi • Thomas G. Chasteen}

(C) Springer-Verlag 2011

The winner of the Hofmeister effect challenge (published in issue 400/3) is

\section{Armando C. Duarte}

CESAM \& Department of Chemistry

University of Aveiro

3810-193 Aveiro, Portugal

The award entitles the winner to select a Springer book of his choice up to a value of $€ 75$.

Our congratulations!

\section{Solution}

Addition of salts to the aqueous phase has two competing effects. First, the extraction efficiency can be boosted by an increase of the concentration of salt - the increased ionic content in the aqueous phase helps to push more of the analyte into the organic phase (salting-out). This advantage, on the other hand, is countered by the fact that the additional salts restrict the migration of the analyte to the extracting phase owing to an increase of the aqueous phase viscosity (salting-in).

Apart from these two effects, in microextraction the presence of salt can change the physical properties of the Nernst diffusion layer of fine organic phase droplets and thus reduce the rate of diffusion of the target analytes into the organic phase (salting-in). As the viscosity of the aqueous phase increases, the movements of the tiny organic solvent droplets are dampened, which, in turn, reduces the rate of mass transfer of the analyte into the organic phase.

In the normal liquid-liquid extraction, salting-out dominates over salting-in. However, in liquid microextraction, this competition is clearly won by the salting-in effects because the extracting organic phase is a fine droplet where any limitations on the diffusion of the analyte have a dramatic effect.

This article is the solution to the Analytical Challenge to be found at http://dx.doi.org/10.1007/s00216-011-4722-z

M. Hashemi $(\triangle)$

Department of Analytical Chemistry, Faculty of Chemistry,

Bu-Ali Sina University,

Hamedan 65178, Islamic Republic of Iran

e-mail: mxh023@shsu.edu

T. G. Chasteen

Department of Chemistry, Sam Houston State University,

Huntsville, TX 77341, USA

e-mail: chm_tgc@shsu.edu 